\title{
COLLECTIVE DYNAMICS IN HEAVY ION COLLISIONS
}

\author{
J.-Y. OLLITRAULT \\ Service de physique théorique, DSM, CEA Saclay
}

\begin{abstract}
These lectures are an elementary introduction to various experimental signatures of collective motion in ultrarelativistic heavy ion collisions: relation between transverse momenta and multiplicities, identified particle spectra, HBT correlations, and elliptic flow.
\end{abstract}

\section{Introduction}

One of the main goals of ultrarelativistic heavy ion collisions is to produce and study the quark-gluon plasma (QGP), a new state of matter where hadrons dissolve into their elementary constituents. One expects a QGP to be formed in these collisions for two reasons: first, a large number of particles are created and seen in detectors; second, due to the Lorentz contraction of the incoming nuclei, the volume of the system shortly after the collision is so small that the density of particles reaches values too high to be compatible with a mere gas of hadrons. Theoretical frameworks to study the quark-gluon plasma, either through perturbative calculations or lattice simulations, assume thermal equilibrium. Now, the system formed in a nucleus-nucleus collision is expanding so rapidly that it can certainly not be described as a static thermal bath. However, the interactions among its constituents may be large enough to achieve thermal equilibrium locally: if this were the case, the dense system formed after the nuclei have passed through each other would behave like an expanding gas, following the laws of fluid dynamics.

Looking for experimental signatures of this (local) thermal equilibrium is therefore a central problem in the phenomenology of heavy ion collisions. I consider it to be an essential preliminary step to the search for the quark-gluon plasma. These lectures are an elementary introduction to this problem. In order to put emphasis on the essential, qualitative ideas behind the various signatures, I have chosen to work out in detail a small number of oversimplified examples, rather than give a general overview of the field. 
Sec. 2 is a short presentation of thermal and hydrodynamical models, based on the idea that the expanding system formed after the collision behaves as an ideal fluid. The strong Lorentz contraction at ultrarelativistic energies leads to a clear separation between longitudinal and transverse degrees of freedom. I will show in particular that an important consequence of thermalization is the longitudinal cooling of the system. Due to this phenomenon, the relation between transverse momenta of outgoing particles and their multiplicity is directly related to the equation of state.

Sec. 3 is devoted to signatures of thermalization in the transverse expansion of the system. Local thermalization would result in a collective motion of the particles superimposed to the thermal motion, as in a flowing liquid. Various signatures of this collective motion are reviewed, based on particle spectra and on two-particle correlations.

Finally, Sec. 4 discusses a phenomenon specific to nucleus-nucleus collisions, namely the correlation between outgoing momenta and the impact direction (or reaction plane) of the two nuclei. This correlation, usually named "anisotropic flow", results solely from interactions between the particles after they have been created in the collision. Therefore, it is widely believed to be the most sensitive probe of collective motion.

\section{Aspects of thermalization}

The collision of two heavy nuclei produces many particles in a small volume. These particles interact. If interactions are strong enough, the mean free path of a particle becomes much smaller than the size of the system: thermalization is reached.

In this ideal limit, the evolution of the system can be described by means of a few parameters. Consider a volume $V$, much smaller than the total volume of the colliding system, yet large enough to contain many particles. In the local rest frame of this subsystem (i.e., the frame where the total momentum of the subsystem vanishes), the momentum (p) distribution of the constituents are isotropic, and their energy $(E)$ distributions are simply given by simple Bose-Einstein or Fermi-Dirac distributions, depending on their spin $s$ :

$$
\frac{d N}{d^{3} \mathbf{x} d^{3} \mathbf{p}}=\frac{(2 s+1)}{(2 \pi)^{3}} \frac{1}{\exp ((E-\mu) / T) \pm 1} .
$$

The only free parameters are the temperature $T$ and a chemical potential $\mu$ (in practice, there is one chemical potential for each conserved quantity, baryon number, electric charge and strangeness).

Momentum distributions in the laboratory frame are then obtained by boosting the system from the local rest frame with a velocity $\beta=$ $\left(\sum \mathbf{p}\right) /\left(\sum E\right)$, where sums run over all constituents of the subsystem. The 
momentum distribution in the laboratory frame is then simply obtained through the following replacement in Eq. (1)

$$
E \rightarrow p^{\mu} u_{\mu}
$$

where $p^{\mu}$ is the four-momentum and $u_{\mu}$ the fluid four-velocity,

$$
u_{\mu}=\frac{1}{\sqrt{1-\beta^{2}}}\left(\begin{array}{c}
1 \\
-\beta
\end{array}\right)
$$

\subsection{CHEMICAL EQUILIBRIUM, KINETIC EQUILIBRIUM}

Thermal equilibrium is a very strong assumption. Eq. (1) indeed constrains not only the momentum distributions, but also the total particle yields, and in particular particle ratios (i.e., relative abundances of various particle species). However, these various aspects of thermal equilibrium are achieved by means of distinct microscopic processes. While all collisions between the particles, either elastic or inelastic, tend to equilibrate momentum distribution, only inelastic collisions may change the number of particles.

Equilibrium with respect to inelastic collisions, which is in some sense the "perfect" thermal equilibrium, is usually referred to as "chemical equilibrium" and will not be studied further in these lectures. It strongly constrains the ratios of particle abundances [1] as well as phase-space densities, which are obtained by combining informations from momentum spectra and two-particle HBT correlations [2], and these constraints seem to be compatible with existing data.

Equilibrium with respect to elastic collisions constrains momentum distributions, and implies in particular that they are isotropic in the local rest frame. This is the "kinetic" equilibrium, on which I concentrate here.

Kinetic equilibrium itself has (at least) two facets. One is the equilibration between longitudinal and transverse degrees of freedom, i.e., the implication that in the local rest frame, longitudinal and transverse momenta are of the same order of magnitude. This aspect of thermalization can be discussed from first principles at the partonic level [3], and there is now a vast literature on this subject [4]. Its major consequence is longitudinal cooling, to be discussed in Sec. 2.3. In order to achieve this "longitudinaltransverse equilibrium", the mean free path of particles must be much smaller than the longitudinal size of the system, much smaller than the nuclear radius due to the strong Lorentz contraction.

However, most of the experimental signatures to be discussed here deal in fact rather with equilibration among the two transverse degrees of freedom. Since the typical transverse scale (the nuclear radius) is much larger 
than the longitudinal scale, this "transverse equilibrium" is probably easier to achieve than "longitudinal-transverse equilibrium".

\subsection{HYDRODYNAMICAL MODELS}

If thermalization is achieved, the evolution of the system is governed by the laws of relativistic fluid dynamics. We need not write the corresponding set of equations here. It is important, however, to understand that they completely determine the evolution of the system from three ingredients:

- An equation of state, i.e., a relation between the pressure, $P$, and the energy density, $\epsilon$.

- Initial conditions, i.e., the specification of the fluid velocity and the energy density on a space-like hypersurface (i.e., at some initial time).

- A "freeze-out" temperature

Initial conditions are of course essential since they fix the geometry of the system. While transverse degrees of freedom are well controlled (the initial transverse fluid velocity is zero by symmetry, while the transverse density profile can be estimated rather accurately from the density of nucleons within the nucleus), longitudinal degrees of freedom (dependence of density on longitudinal coordinates, longitudinal fluid velocity) are to a large extent arbitrary.

Finally, the freeze-out temperature is a crude way of parameterizing the smooth transition from a thermalized state to a free-streaming regime: when the mean free path becomes as large as the system, which occurs approximately at a given temperature (the freeze-out temperature $T_{f}$ ), one assumes that particles follow straight lines to the detectors. One must keep in mind that this really is a rough approximation. If the mean free path at temperature $T_{f}$ is equal to the system size, it is obvious that the mean free path at a temperature, say, $1.5 T_{f}$ cannot be much smaller than the system size, so that hydrodynamics at temperatures not much larger than $T_{f}$ cannot hold strictly.

\subsection{LONGITUDINAL COOLING}

Due to the strong Lorentz contraction, the primary collisions between nucleons essentially occur at $z=t=0$, where $z$ is the collision axis. The longitudinal size of the system then grows proportional to $t$, and the early stages of the evolution are dominated by this longitudinal expansion. Now, if the system is thermalized, it cools during the expansion: the energy $E$ contained in a given fluid element decreases as its volume $V$ increases since $d E=-P d V$. This is a nontrivial phenomenon. It is interesting to note 
that models of initial conditions based on minijet production do require a substantial amount of cooling in order to reproduce observables [5].

The cooling is effectively cut off by the transverse expansion of the system (whether or not the system is in equilibrium): the total energy is effectively frozen at a time of the order of half the transverse radius for a cylindrically symmetric source [6]. The energy density at this time can be estimated from the final transverse energy of particle multiplicity. On the other hand, average transverse momenta of outgoing particles are related to the energy per particle, which is given by the equation of state. Therefore, it is a nontrivial prediction of hydrodynamical models that they can reproduce simultaneously particle multiplicities and transverse momentum spectra. Without longitudinal cooling, such agreement is much harder to reach [7].

\subsection{TRANSVERSE COLLECTIVE FLOW}

Initially, the fluid transverse velocity is zero by symmetry. Transverse collective motion is created by pressure gradients. For a nonrelativistic system, the fluid acceleration is given by Euler's equation:

$$
\frac{d \beta}{d t}=-\frac{1}{\rho} \nabla P,
$$

with $\rho$ the mass density. The relativistic generalization of this equation is

$$
\frac{d \beta}{d t}=-\frac{c^{2}}{\epsilon+P} \nabla P
$$

with $\epsilon$ the total energy density. Time scales associated with transverse collective flow are thus naturally of the order of the transverse size of the system, which gives rise to the gradients: any observable associated with transverse collective flow ( $p_{T}$ spectra, HBT correlations, elliptic flow...) probes the system on a time scale which is essentially of the order of the transverse radius.

\section{Various one- and two-particle observables}

In this section, I discuss various observables which are commonly used in order to characterize the transverse collective expansion of the system. More precisely, one tries to extract from experimental data a quantitative information about the freeze-out temperature, $T$, and the transverse collective velocity, $\beta_{\perp}$, at freeze-out. The discussion will remain at a very elementary level. The goal here is simply to grasp simple, but essential qualitative ideas.

I will first show that transverse momentum spectra of various identified particles can help to disentangle thermal and collective motion. I then turn 
to signatures of space-momentum correlations, which may be more specific probes of collective motion: quantum correlations between identical particles, the so-called Hanbury-Brown and Twiss (HBT) effects, yield direct information on the size of the emitting source; the production of deuterons and light nuclei, which involves the phase space density (i.e., the density in momentum and space) of the particles, is another probe of space-momentum correlations, which will not be studied here.

\subsection{SINGLE PARTICLE $P_{T}$-SPECTRA}

The four-momentum of a particle with mass $m$ is usually parameterized in the form

$$
p^{\mu}=\left(\begin{array}{l}
E=m_{T} \cosh y \\
p_{z}=m_{T} \sinh y \\
\mathbf{p}_{\mathbf{T}}
\end{array}\right),
$$

where $y$ is the rapidity of the particle, $\mathbf{p}_{\mathbf{T}}$ its transverse momentum and $m_{T}$ its transverse mass. The mass-shell condition $p^{\mu} p_{\mu}=m^{2}$ gives the relation $m_{T}^{2}=p_{T}^{2}+m^{2}$.

In a two-dimensional transverse world $\left(p_{z}=0\right)$, the energy $E$ coincides with the transverse mass $m_{T}$. For a thermalized fluid at rest, neglecting effects of quantum statistics, transverse momentum distributions follow a Boltzmann distribution:

$$
\frac{d N}{d^{2} \mathbf{p}_{\mathbf{T}}} \propto e^{-m_{T} / T}
$$

If one takes into account the longitudinal momentum, the exponential is replaced by a more complicated expression involving Bessel functions, which essentially differ from the exponential behavior by powers of $m_{T}$.

Surprisingly, it turns out that transverse momentum distributions of particles produced in heavy ion collisions, as well as in elementary processes, are rather well fit by such simple exponentials in $m_{T}$. If the system undergoes collective transverse expansion, however, the "inverse slope parameter" $T$ obtained by fitting $p_{T}$ spectra no longer corresponds to the temperature of the fluid. We therefore denote this parameter by $T_{\text {eff }}$ (effective temperature).

In order to understand how the inverse slope parameter $T_{\text {eff }}$ is related to the temperature $T$ and to the fluid velocity $\beta_{\perp}$, we consider for simplicity a one-dimensional, non-relativistic fluid. The velocity $v$ of a particle in the fluid is the sum of a random, thermal component, $v^{*}$, and the fluid velocity $\beta$ :

$$
v=v^{*}+\beta \text {. }
$$


The average kinetic energy for a particle of mass $m$ is therefore

$$
\left\langle\frac{m v^{2}}{2}\right\rangle=\left\langle\frac{m\left(v^{*}\right)^{2}}{2}\right\rangle+\frac{m \beta^{2}}{2},
$$

where the cross term vanishes due to the random orientation of the thermal velocity $v^{*}$. Now, the thermal energy is simply related to the temperature: $\left\langle m(v *)^{2}\right\rangle / 2=T / 2$ for a one dimensional fluid, while the average kinetic energy is similarly related to the "effective temperature" $T_{\text {eff }}$. One thus obtains

$$
T_{\text {eff }}=T+m \beta^{2} .
$$

Coming back to transverse momentum spectra in nucleus-nucleus collision, one may derive a similar relation, with $\beta$ replaced by the transverse fluid velocity $\beta_{\perp}$. [8]

Two important lessons can be drawn from this equation. First, one cannot easily disentangle effects of thermal motion and collective motion from the spectra of a single species of particles: one only sees the inverse slope (10) which is a combination of both. This can be seen in Fig. 1 which displays constraints on $T$ and $\beta_{\perp}$ from a thermal fit to negative hadron spectra. For small $\beta_{\perp}$ (don't forget that Eq. (10) only holds for a nonrelativistic fluid), the allowed region indeed follows a curve of the type $T+m \beta_{\perp}^{2}=$ constant. Second lesson, the inverse slope parameter $T_{\text {eff }}$ increases with particle mass when collective expansion is present, so that collective expansion and thermal motion may be disentangled by comparing spectra of different particles. This is also seen in Fig. 1: deuterium, which is heavier, is more sensitive to the collective fluid velocity than other negative hadrons.

To conclude this section, let us mention that other mechanisms might produce inverse slopes which increase with mass [10], so that single-particle spectra alone cannot provide conclusive evidence for transverse collective flow.

\subsection{QUANTUM CORRELATIONS}

Correlations of identical particles, in particular pions, provide direct information on the size of the emitting source. A huge number of papers, both experimental and theoretical (see [11] for reviews) are devoted to this rather technical issue, which I merely illustrate by means of a few simple examples.

\subsubsection{Basics of $H B T$}

Let us first briefly recall the standard derivation of the effect for a static system: two identical pions are emitted from a source (sketched in Fig. 2) 


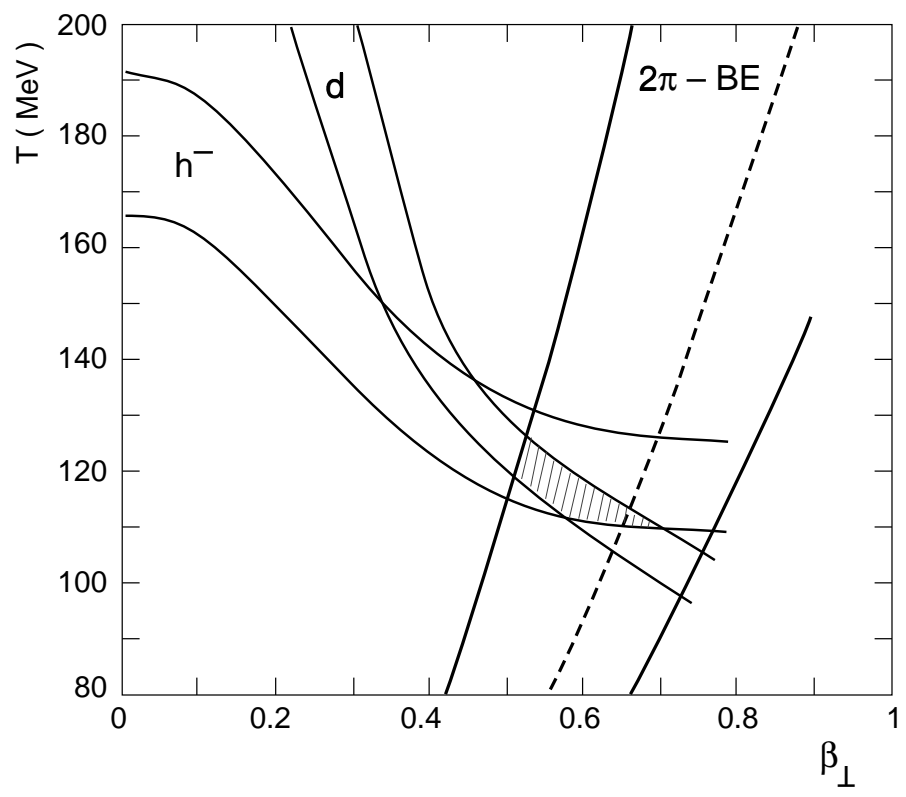

Figure 1. Thermal fits to negative hadron $p_{T^{-}}$-spectra $\left(h^{-}\right)$, deuteron $p_{T}$-spectra $(d)$ and $\mathrm{HBT}$ radii $(2 \pi$-BE) for $\mathrm{Pb}-\mathrm{Pb}$ collisions at $158 \mathrm{GeV}$ per nucleon, analyzed by the NA49 Collaboration (from [9]).

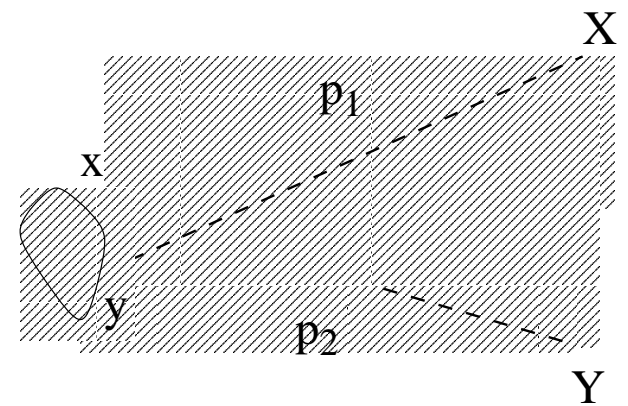

Figure 2. Schematic representation of the quantum interference between identical pions: their two possible trajectories from the source to the detector, which are indistinguishable in quantum mechanics, are represented by full and dashed lines.

at points $\mathbf{x}$ and $\mathbf{y}$, and detected at points $\mathbf{X}$ and $\mathbf{Y}$, respectively. The wave function of the two pions at the point where they are produced is

$$
\psi(\mathbf{x}, \mathbf{y}) \propto e^{i \mathbf{p}_{1} \cdot \mathbf{x}} e^{i \mathbf{p}_{2} \cdot \mathbf{y}},
$$

where $\mathbf{p}_{\mathbf{1}}$ and $\mathbf{p}_{\mathbf{2}}$ are the momenta of the pions detected at $\mathbf{X}$ and $\mathbf{Y}$, respectively. If the two pions are identical, their wave function must be symmetric, i.e., $\psi(\mathbf{x}, \mathbf{y})=\psi(\mathbf{y}, \mathbf{x})$. Symmetrizing the above expression, one 
obtains

$$
\psi(\mathbf{x}, \mathbf{y}) \propto \frac{1}{\sqrt{2}}\left(e^{i \mathbf{p}_{\mathbf{1}} \cdot \mathbf{x}} e^{i \mathbf{p}_{\mathbf{2}} \cdot \mathbf{y}}+e^{i \mathbf{p}_{\mathbf{1}} \cdot \mathbf{y}} e^{i \mathbf{p}_{\mathbf{2}} \cdot \mathbf{x}}\right),
$$

where the second term corresponds to the trajectories represented as dashed lines in Fig. 2, obtained by exchanging $\mathbf{X}$ and $\mathbf{Y}$. (Note that the source size is much, much smaller than the distance to the detector, so that the direction of the momentum of a particle seen at a given in the detector does not depend on the point where it was emitted).

The probability is obtained by squaring the above amplitude, which yields an interference term:

$$
|\psi(\mathbf{x}, \mathbf{y})|^{2} \propto 1+\operatorname{Re}\left(e^{i\left(\mathbf{p}_{1}-\mathbf{p}_{2}\right) \cdot \mathbf{x}} e^{-i\left(\mathbf{p}_{1}-\mathbf{p}_{2}\right) \cdot \mathbf{y}}\right) .
$$

If $\rho(\mathbf{x})$ denotes the density of the source normalized to unity, averaging the above expression over $\mathbf{x}$ and $\mathbf{y}$ with the weight $\rho(\mathbf{x}) \rho(\mathbf{y})$, one obtains the two particle correlation function

$$
C_{2}\left(\mathbf{p}_{\mathbf{1}}, \mathbf{p}_{\mathbf{2}}\right) \equiv \frac{f\left(\mathbf{p}_{\mathbf{1}}, \mathbf{p}_{\mathbf{2}}\right)}{f\left(\mathbf{p}_{\mathbf{1}}\right) f\left(\mathbf{p}_{\mathbf{2}}\right)}=1+\left|\int d^{3} \mathbf{x} \rho(\mathbf{x}) e^{i\left(\mathbf{p}_{1}-\mathbf{p}_{2}\right) \cdot \mathbf{x}}\right|^{2},
$$

where $f\left(\mathbf{p}_{\mathbf{1}}, \mathbf{p}_{\mathbf{2}}\right)$ denotes the two-body momentum-space density.

The scaled correlation $C_{2}\left(\mathbf{p}_{\mathbf{1}}, \mathbf{p}_{\mathbf{2}}\right)$ is therefore directly related to the Fourier transform of the source density at the point $\mathbf{p}_{\mathbf{1}}-\mathbf{p}_{\mathbf{2}}$. The Fourier transform typically has a bell shape, and decrease from 1 for $\mathbf{p}_{\mathbf{1}}-\mathbf{p}_{\mathbf{2}}=0$, down to 0 for large $\left|\mathbf{p}_{\mathbf{1}}-\mathbf{p}_{\mathbf{2}}\right|$, with a typical width of $1 / R$, where $R$ is the source size, the so-called "HBT radius".

The final question is: what is the "source" in the case of a heavy ion collision? Here, the system is no longer static, and its expansion must be taken into account. It is believed that HBT radii reflect the position of the pions when they "freeze-out", i.e., when they scatter for the last time. Typical HBT radii are of order $R \sim 4 \mathrm{fm}$, thus the correlation due to the quantum interference is sizable only for relative momenta less than $1 / R \sim 50 \mathrm{MeV} / \mathrm{c}$, much smaller than the average transverse momentum of a pion, roughly $400 \mathrm{MeV} / \mathrm{c}$. Therefore, quantum correlations are short-range correlations in momentum space.

\subsubsection{Collective motion reduces $H B T$ radii}

We have assumed above that the density of the source depends on the position $\mathbf{x}$ only. Within a flowing system, however, there are space-momentum correlations, so that the density also depends on momenta. In practice, the above analysis can be carried out for a fixed value of the average momentum $\left(\mathbf{p}_{1}+\mathbf{p}_{2}\right) / 2$. Since the relative momenta $\mathbf{p}_{1}-\mathbf{p}_{\mathbf{2}}$ of interest are small, this amounts to fixing the value of $\mathbf{p}_{\mathbf{1}}$. Therefore, the HBT radius given by the 
analysis is the size of the source that emits pions with a given momentum $\mathbf{p}_{1}$.
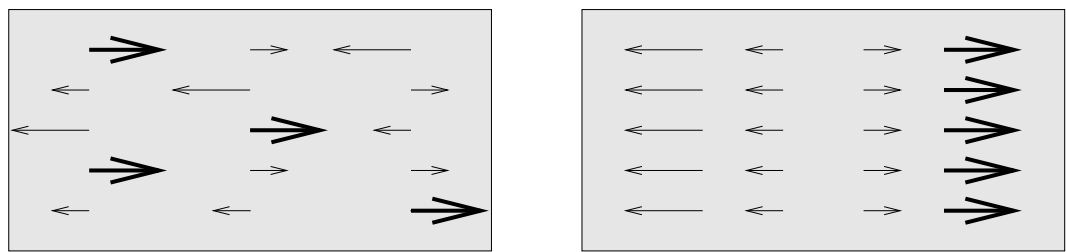

Figure 3. Schematic picture of two one-dimensional sources, of equal length $R$. Left: disordered source; right: perfectly ordered source.

One then easily understands how HBT radii may reflect collective motion in the system. For this purpose, we consider a simplified, one-dimensional world depicted in Fig. 3. If there is no collective motion, space and momentum coordinates are uncorrelated, and the HBT radius is the true width of the source, $R$ (Fig. 3, left). In the case when the motion is purely collective (no thermal dispersion), on the other hand, there is a one-to-one correspondence between momentum and space coordinates (Fig. 3, right), so that all particles with a given momentum originate from the same point. In this extreme case, the HBT radius vanishes. One understands from this simple picture that collective motion generally results in smaller HBT radii. Indeed, HBT radii measured in $\mathrm{Pb}-\mathrm{Pb}$ collisions are not much larger than in collisions with lighter projectiles, and significantly smaller than the radius of the $\mathrm{Pb}$ nucleus.

\subsubsection{Longitudinal expansion and $m_{T}$ scaling of longitudinal radii}

Sizable space-momentum correlations are naturally expected in the longitudinal direction, whether or not thermal equilibrium is achieved. Indeed, we have seen that due to the strong Lorentz contraction, primary nucleonnucleon collisions essentially occur at the origin $z=t=0$. If a particle is issued from this point without subsequent rescattering, its longitudinal velocity is exactly given by $v_{z}=z / t$ : space and momentum are strictly correlated for a free-streaming system, as in Fig. 3, right! If thermalization occurs, a random thermal motion will destroy part of this correlation, but it is reasonable to assume that the average (fluid) longitudinal velocity is indeed given by $\beta_{z}=z / t[12]$.

Let us now compute the source density for particles with a given momentum in this longitudinally expanding fluid. For simplicity, we approximate Eq. (1) by a Boltzmann distribution. For a fixed momentum $p^{\mu}$, the spatial density is given by

$$
\frac{d N}{d^{3} \mathbf{x}} \propto \exp \left(-\frac{p^{\mu} u_{\mu}}{T}\right)
$$


For a particle emitted at zero rapidity, Eq. (6) gives $p^{\mu}=\left(m_{T}, 0, \mathbf{p}_{\mathbf{T}}\right)$. Neglecting the transverse fluid velocity, Eq. (3) gives $u_{\mu}=(1,-z / t, 0) / \sqrt{1-z^{2} / t^{2}}$. One thus obtains the source density:

$$
\frac{d N}{d z} \propto \exp \left(-\frac{m_{T}}{T \sqrt{1-z^{2} / t^{2}}}\right)
$$

where $t$ is the time at which the system decouples. If $m_{T}$ is significantly larger than the temperature $T$, one can expand the exponent to leading order in $z$ :

$$
\frac{d N}{d z} \propto \exp \left(-\frac{m_{T}}{2 T} \frac{z^{2}}{t^{2}}\right) .
$$

This is a gaussian profile, with a longitudinal width

$$
R_{\text {long }}=t \sqrt{\frac{T}{m_{T}}} .
$$

This leads to the prediction that longitudinal HBT radii scale like $1 / \sqrt{m_{T}}$ in a thermalized fluid [13], a prediction indeed supported by data. Whether or not this $m_{T}$ scaling constitutes a strong evidence for thermalization is left to the appreciation of the reader.

\subsubsection{Transverse radii and transverse collective flow}

Similarly, the $m_{T}$ dependence of the source transverse size can be used to constrain parameters of thermal models, namely, the temperature $T$ and the fluid velocity $\beta_{\perp}$. For simplicity, we again consider a one-dimensional, nonrelativistic model. We assume that the source has a gaussian density profile with radius $R$ :

$$
\rho(x) \propto \exp \left(-\frac{x^{2}}{2 R^{2}}\right) .
$$

In order to mimic the transverse expansion in a heavy ion collision, we further assume that the source is expanding symmetrically away from the origin, with a simple fluid velocity profile:

$$
\beta(x)=\beta_{\perp} \frac{x}{R},
$$

where $\beta_{\perp}$ is a constant, which represents the typical velocity of the fluid. The density in coordinate $(x)$ and velocity $(v)$ space is then given by

$$
\frac{d N}{d x d v} \propto \exp \left(-\frac{E^{*}}{T}\right) \rho(x)
$$


where $E^{*}$ is the kinetic energy in the rest frame of the fluid, i.e.,

$$
E^{*}=m(v-\beta(x))^{2} / 2,
$$

where $\beta(x)$ is given by Eq. (20) Inserting Eqs. (19) and Eq. (22) into Eq. (21), one obtains for fixed $v$ the following density profile

$$
\frac{d N}{d x} \propto \exp \left(-\frac{x^{2}}{2 R^{2}}\left(\frac{m \beta_{\perp}^{2}}{T}+1\right)\right)
$$

This is again a gaussian, with radius

$$
R_{\mathrm{HBT}}=\frac{R}{\sqrt{1+m \beta_{\perp}^{2} / T}} .
$$

As expected, collective expansion reduces the HBT radius.

For a relativistic fluid, one obtains a similar expression, where $m$ is replaced by the transverse mass $m_{T}$ : transverse HBT radii also decrease with transverse mass. From the observed decrease, one may constrain the parameter $\beta_{\perp}^{2} / T$. This is illustrated in Fig. 1: the constraint from HBT correlations indeed follows a curve of the type $\beta_{\perp}^{2} / T=$ constant. Remarkably, the values of $\beta_{\perp}$ and $T$ obtained from single-particle spectra automatically satisfy this new constraint from HBT correlations.

\section{Elliptic flow and multiparticle correlations}

The phenomenon of elliptic flow is widely considered as the most direct evidence that final state interactions, driving the colliding system towards local thermal equilibrium, are indeed large in nucleus-nucleus collisions. I first give a general definition of azimuthal anisotropies, to which elliptic flow belongs; then I show that elliptic flow is a sensitive probe of thermalization; finally, I discuss how elliptic flow is obtained experimentally: this subtle issue indeed deserves some interest.

\subsection{DIRECTED AND ELLIPTIC FLOW: DEFINITIONS}

In a non-central nucleus-nucleus collision, the impact parameter defines a reference direction in the transverse plane. One usually calls "reaction plane" the plane spanned by the impact parameter and the collision axis. It turns out that azimuthal angles of outgoing particles are most often correlated to this reference direction. This is the phenomenon of anisotropic flow. If $\phi$ denotes the azimuthal angle of a particle with respect to the 


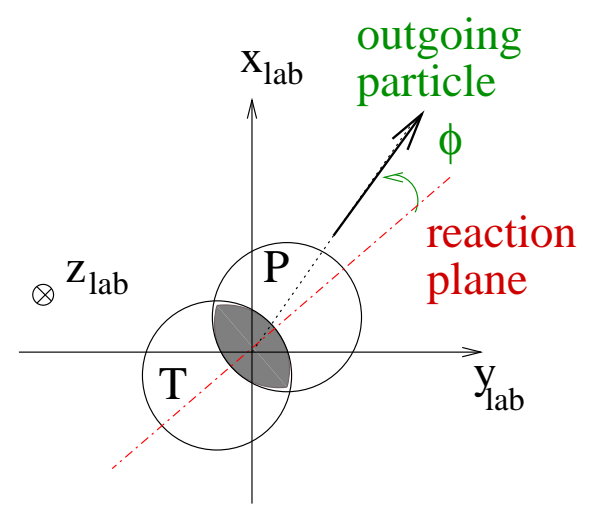

Figure 4. Nucleus-nucleus collisions viewed in the transverse plane. $x_{\text {lab }}$ and $y_{\text {lab }}$ are fixed directions in the laboratory system. The dash-dotted line is the direction of impact parameter, or reaction plane.

reaction plane (see Fig. 4), such a correlation means that the $\phi$ distribution is not flat. The latter is usually expanded in Fourier series [14]

$$
\frac{d N}{d \phi} \propto 1+2 \sum_{n=1}^{\infty} v_{n} \cos (n \phi),
$$

where terms proportional to $\sin (n \phi)$ vanish due to the $\phi \rightarrow-\phi$ symmetry. The Fourier coefficients $v_{n}$ characterize the strength of anisotropic flow:

$$
v_{n}=\left\langle e^{i n \phi}\right\rangle
$$

where brackets denote a statistical average. The first two Fourier coefficients $v_{1}$ and $v_{2}$ are usually called "directed flow" and "elliptic flow", and have been measured at various colliding energies, from below $50 \mathrm{MeV}$ per nucleon up to RHIC energies. [15]

This phenomenon is of crucial importance for the following reason: if the nucleus-nucleus collision was a mere superposition of independent nucleonnucleon collisions, the $\phi$ distribution would be flat: a pair of colliding nucleons does not see the impact parameter of the whole nucleus-nucleus collision. For this reason, anisotropies in the $\phi$ distribution must result from final state interactions of the produced particles. This is illustrated below, where we discuss a mechanism that produces elliptic flow at ultrarelativistic energies.

\subsection{THE PHYSICS OF ELLIPTIC FLOW}

At ultrarelativistic energies, the azimuthal anisotropy is dominated by elliptic flow, and $v_{2}$ is positive. This can be easily understood. A large number of 


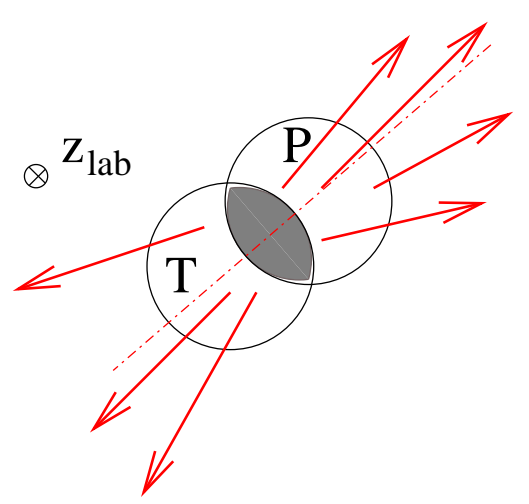

Figure 5. Typical directions of outgoing particles at ultrarelativistic energies.

particles are created in an almond-shaped region, represented by the shaded area in Fig. 5. Interactions between these particles result in a pressure which is highest at the center of the almond, and zero outside. At a given point, the resulting force per unit volume is opposite to the pressure gradient, see Eq. (5). Now, the gradient is larger along the smaller direction of the almond, which is precisely the direction of the reaction plane. Thus one expects stronger collective motion in the direction of the reaction plane than in the perpendicular direction. This results in a positive value of $v_{2}$, which was predicted in [16] and later observed at the top AGS energy [17] and at SPS [9].

\subsection{CENTRALITY DEPENDENCE OF ELLIPTIC FLOW}

Hydrodynamical models are able to provide stable, quantitative predictions for this effect. Indeed, what matters here is essentially the shape of the almond, i.e., the distribution of energy in the transverse plane, which is well controlled theoretically. On the other hand, $v_{2}$ depends weakly on the scenario chosen for the longitudinal expansion, which is to a large extent arbitrary as discussed in Sec. 2. It turns out that the resulting $v_{2}$ always decreases linearly [16] with centrality (as estimated from the total charged multiplicity or transverse energy produced in the collision). This is illustrated in Fig. 6. The absolute magnitude on $v_{2}$, however, depends on the equation of state. More precisely, it increases with the velocity of sound $c_{s} \equiv \sqrt{d P / d \epsilon}$, as one would expect intuitively: a higher pressure produces more $v_{2}$.

The centrality dependence of elliptic flow therefore yields valuable information about the degree of thermalization achieved in the system[20]. If thermalization is only partial, departures from thermalization (which yield smaller values of elliptic flow) are expected to be more significant for the 


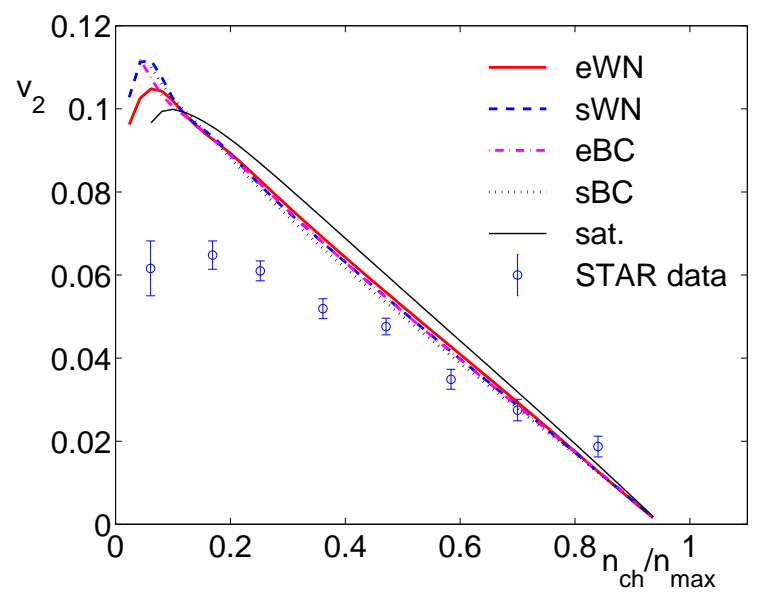

Figure 6. Elliptic flow as a function of centrality for a Au-Au collision at RHIC. The curves are predictions of a hydrodynamic model, for various choices of initial conditions. From P. Kolb et al. [19].

more peripheral collisions: the size of the system is smaller, so that particles undergo fewer collisions. Then, the maximum of $v_{2}$ occurs at less peripheral collisions than if thermalization if fully achieved. This is indeed observed in all calculations done with transport models $[21,22]$ which contain final state interactions but do not assume perfect thermalization. This is illustrated in Fig. 7.

\section{4. $P_{T}$ DEPENDENCE OF ELLIPTIC FLOW}

A great success of thermal models is that they also naturally reproduce the non-trivial $p_{T}$ dependence of elliptic flow for various identified particles [23]. This is illustrated in Fig. 8. However, a saturation of elliptic flow is observed for $p_{T}$ above $2 \mathrm{GeV}$ (see Fig. 11), which is not reproduced by hydrodynamical calculations. This phenomenon has been proposed as a possible signature of jet quenching [25].

\subsection{FLOW ANALYSIS}

Measuring elliptic flow, and more generally anisotropic flow, is far from obvious. Indeed, the orientation of the reaction plane is unknown experimentally, so that the azimuthal angle $\phi$ defined in Fig. 4 is not a measurable quantity, and elliptic flow defined by Eq. (26) is also not directly measurable. Only relative azimuthal angles can be measured experimentally. 


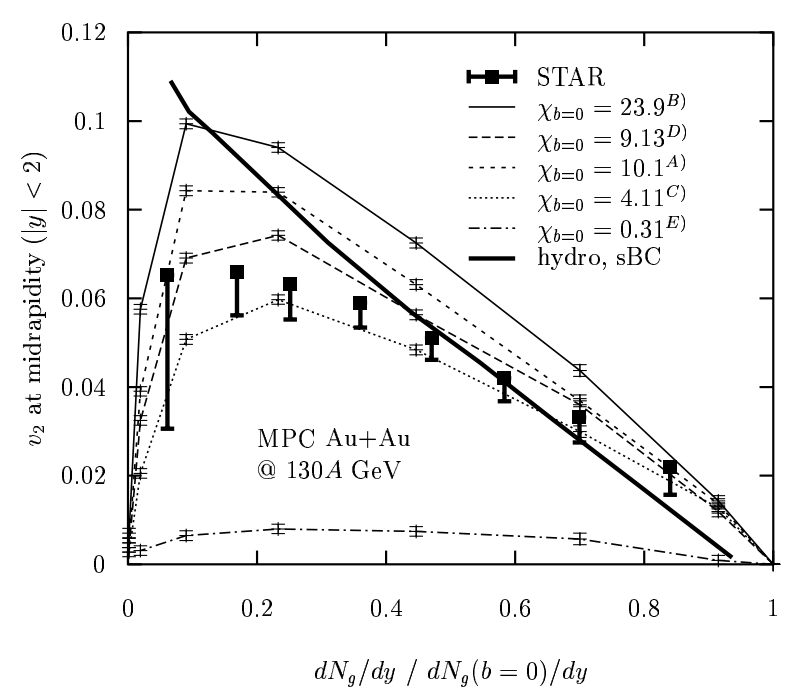

Figure 7. Predictions from a transport calculation. The strength of final state interactions increases from bottom to top. As interactions increase, one gets closer to the hydrodynamical limit, and the maximum of $v_{2}$ correspondingly shifts towards more peripheral collisions, From D. Molnar and M. Gyulassy [22].

\subsubsection{Standard analysis}

The standard flow analysis [26] relies on the key assumption that particles are independent. This allows one to write [27]:

$$
\left\langle e^{2 i\left(\phi_{1}-\phi_{2}\right)}\right\rangle=\left\langle e^{2 i \phi_{1}}\right\rangle\left\langle e^{-2 i \phi_{2}}\right\rangle=\left(v_{2}\right)^{2},
$$

where brackets denote an average over pairs of particles belonging to the same event. From the measured two-particle average in the left-hand side, one thus obtains the elliptic flow $v_{2}$, up to a sign.

\subsubsection{Beware of nonflow correlations!}

However, the above equation is not quite correct, since it neglects correlations between particles. Such correlations generally produce an additional term in the right-hand side of Eq. (27). The magnitude of a two-particle correlation generally scales with the total number of particles $N$ like $1 / N$, and this is generally the order of magnitude of the additional, "nonflow" term:

$$
\left\langle e^{2 i\left(\phi_{1}-\phi_{2}\right)}\right\rangle=\left(v_{2}\right)^{2}+\mathcal{O}(1 / N)
$$

At SPS energies, $N \simeq 2500$ for a central $\mathrm{Pb}-\mathrm{Pb}$ collision, while $v_{2}$ is of the order of $3 \%$ : both terms in the right-hand side of Eq. (28) are of the same order, and one may no longer ignore nonflow correlations. Similar arguments apply to directed flow $v_{1}$. 


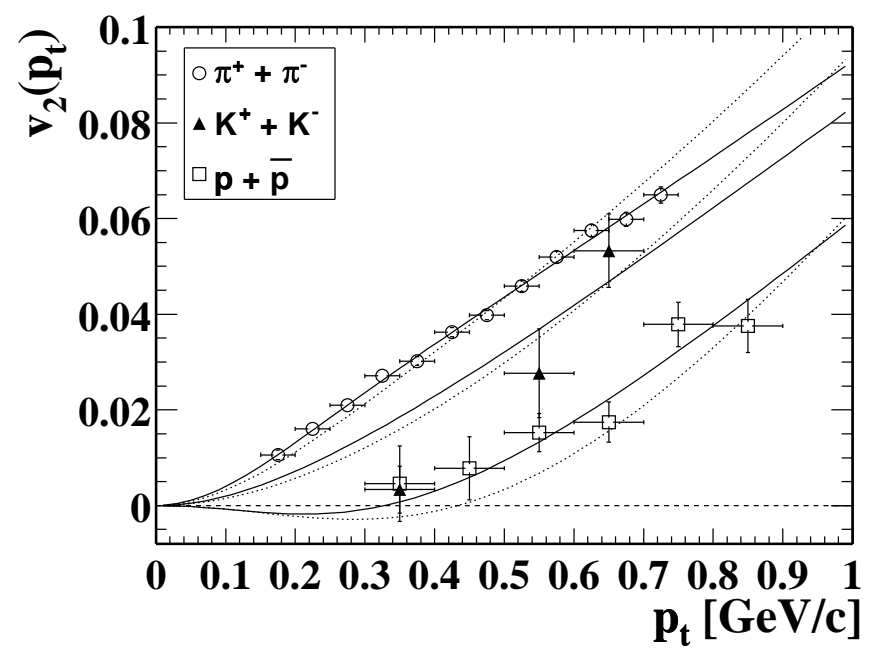

Figure 8. $\quad p_{T}$ dependence of elliptic flow for pions, kaons and protons, measured by the STAR Collaboration at RHIC, together with predictions from a thermal model, from [24].

Several sources of correlations between particles are well known, and their contribution to the last term of Eq. (28) can be estimated. For example, the quantum correlations already studied in Sec. 3.2 do produce sizable azimuthal correlations between particles with low relative momenta. Taking this effect into account, one is led to revise significantly the values of the flow given by the standard analysis. This is illustrated in Fig. 9 which shows the corresponding modification in the case of pion directed flow. Correlations due to global momentum conservation also lead to significant corrections.

\subsubsection{Cumulants of multiparticle correlations}

The contribution of nonflow correlations can be greatly reduced by going beyond two-particle methods. One may for instance construct a four-particle average such as $\left\langle e^{2 i\left(\phi_{1}+\phi_{2}-\phi_{3}-\phi_{4}\right)}\right\rangle$, where $\phi_{1}, \cdots, \phi_{4}$ are azimuthal angles of four particles belonging to the same events. Repeating the argument leading to Eq. (27), this quantity is also related to elliptic flow:

$$
\left\langle e^{2 i\left(\phi_{1}+\phi_{2}-\phi_{3}-\phi_{4}\right)}\right\rangle=\left(v_{2}\right)^{4}
$$

but the corresponding estimate of $v_{2}$ is also biased by nonflow correlations.

However, the contribution of nonflow correlations can be greatly reduced by combining the informations from two- and four-particle averages, Eqs. (27) and (29). Indeed, let us assume that particles are pairwise correlated. Then, the four-particle average can be written as a sum of two 


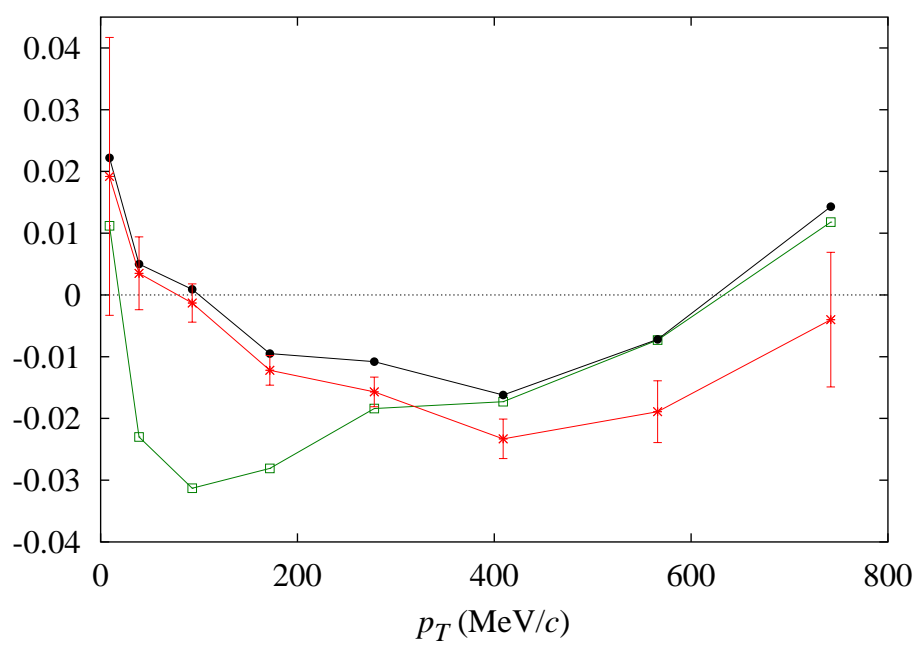

Figure 9. Directed flow of pions in $\mathrm{Pb}-\mathrm{Pb}$ collisions at $158 \mathrm{GeV}$ per nucleon. Open squares: result of the standard flow analysis performed by NA49 [9]; full squares: after subtraction of HBT correlations; stars: after subtraction of correlations from global momentum conservation (from [28]).

terms:

$$
\left\langle e^{2 i\left(\phi_{1}+\phi_{2}-\phi_{3}-\phi_{4}\right)}\right\rangle=\left\langle e^{2 i\left(\phi_{1}-\phi_{3}\right)}\right\rangle\left\langle e^{2 i\left(\phi_{2}-\phi_{4}\right)}\right\rangle+\left\langle e^{2 i\left(\phi_{1}-\phi_{4}\right)}\right\rangle\left\langle e^{2 i\left(\phi_{2}-\phi_{3}\right)}\right\rangle .
$$

The first term in the right-hand side corresponds to the situation where particles 1 and 3 form one pair and particles 2 and 4 a second pair, while the second term corresponds to the second possibility, 1 with 4 and 2 with 3 (the third possibility, namely 1 with 2 and 3 with 4 , gives a vanishing contribution). If averages are taken over all possible 4-uplets of particles, this equation becomes simply

$$
\left\langle e^{2 i\left(\phi_{1}+\phi_{2}-\phi_{3}-\phi_{4}\right)}\right\rangle=2\left\langle e^{2 i\left(\phi_{1}-\phi_{3}\right)}\right\rangle^{2} .
$$

Subtracting the right-hand side from the left-hand side, one therefore obtains a quantity which is free from two-particle correlations. This is the cumulant of the four-particle correlation. Quite remarkably, the cumulant no longer vanishes if elliptic flow is present. It thus yields an estimate of $v_{2}$, easily obtained by combining Eqs. (27) and (29):

$$
\left\langle e^{2 i\left(\phi_{1}+\phi_{2}-\phi_{3}-\phi_{4}\right)}\right\rangle-2\left\langle e^{2 i\left(\phi_{1}-\phi_{2}\right)}\right\rangle^{2}=-\left(v_{2}\right)^{4},
$$

and this estimate is essentially free from nonflow correlations. [29] 


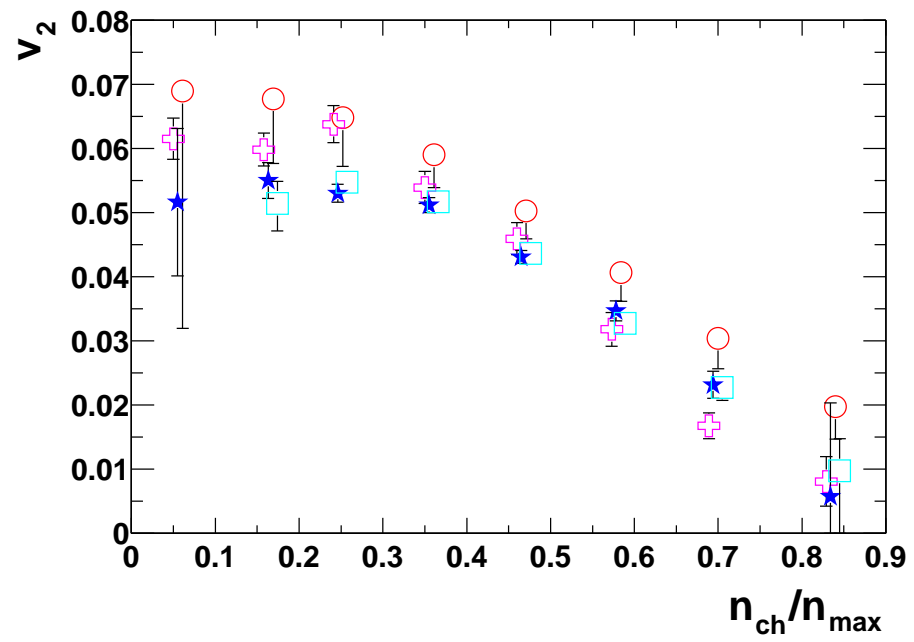

Figure 10. Elliptic flow versus centrality. Circles: from the standard, two-particle analysis; stars: from the cumulant of four-particle correlations (from [31]).

This method was recently applied to STAR data $[30,31]$. The corresponding centrality dependence of elliptic flow is displayed in Fig. 10. The values of $v_{2}$ from cumulants of four-particle correlations are significantly smaller than those obtained with the standard flow analysis, in particular for the most peripheral collisions. this is precisely where nonflow effects are expected to give the largest contribution since the multiplicity $N$ is smaller (see Eq. (28). The centrality dependence obtained with this method suggests that departures from thermalization at RHIC may be larger than was previously thought.

The $p_{T}$ dependence of elliptic flow has also be analyzed with the fourparticle method. The corresponding results are displayed in Fig. 11. Deviations from the standard method seem to be larger at high $p_{T}$. The corresponding nonflow correlations may be due to minijets, which produce high $p_{T}$ particles with azimuthal angles strongly correlated[32]

This cumulant expansion can be worked out to arbitrary orders, and allows one to extract the genuine 4-, 6-particle correlations and beyond.[29] Flow, which is essentially a collective phenomenon, contributes to all orders, while the relative contribution of nonflow correlations decreases as the order increases. Higher order cumulants are therefore a unique tool to check quantitatively that azimuthal correlations are indeed of collective origin. 


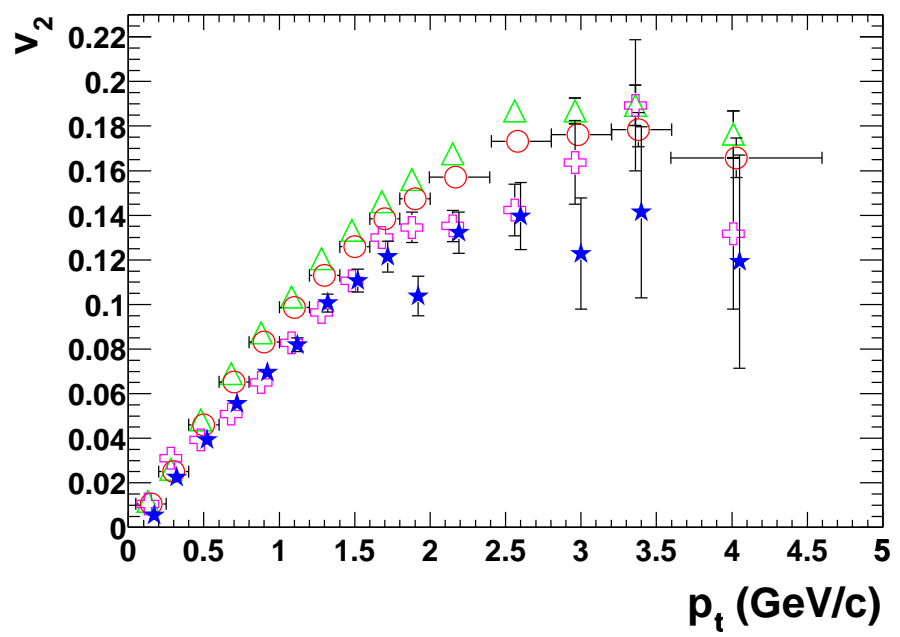

Figure 11. Elliptic flow of charged particles as a function of transverse momentum, analyzed by the STAR Collaboration at RHIC. Triangles and circles: from two-particle analyses; stars: from the cumulant of four-particle correlations (from [31]).

\section{References}

1. F. Becattini, M. Gaździcki and J. Sollfrank, Eur. Phys. J. C 5, 143 (1998); P. BraunMunzinger, I. Heppe and J. Stachel, Phys. Lett. B 465, 15 (1999).

2. G. F. Bertsch, Phys. Rev. Lett. 72, 2349 (1994) [Erratum-ibid. 77, 789 (1996)]; D. Ferenc et al., Phys. Lett. B 457, 347 (1999).

3. E. V. Shuryak, Phys. Rev. Lett. 68, 3270 (1992).

4. See for instance R. Baier, A. H. Mueller, D. Schiff and D. T. Son, Phys. Lett. B 502, 51 (2001) and references therein.

5. K. J. Eskola, H. Niemi, P. V. Ruuskanen and S. S. Räsänen, hep-ph/0206230.

6. J.-Y. Ollitrault, Phys. Lett. B 273, 32 (1991).

7. U. W. Heinz and S. M. Wong, Phys. Rev. C 66 (2002) 014907

8. E. Schnedermann, J. Sollfrank and U. W. Heinz, Phys. Rev. C 48, 2462 (1993); I. G. Bearden et al. [NA44 Collaboration], Phys. Rev. Lett. 78, 2080 (1997).

9. H. Appelshäuser et al. [NA49 Collaboration], Eur. Phys. J. C 2, 661 (1998).

10. J. Schaffner-Bielich et al., nucl-th/0108048.

11. U. A. Wiedemann and U. W. Heinz, Phys. Rept. 319 (1999) 145; R. M. Weiner, Phys. Rept. 327 (2000) 249.

12. J. D. Bjorken, Phys. Rev. D 27, 140 (1983).

13. A. N. Makhlin and Y. M. Sinyukov, Z. Phys. C 39 (1988) 69.

14. S. Voloshin and Y. Zhang, Z. Phys. C 70, 665 (1996).

15. For a short review, see J. Y. Ollitrault, Nucl. Phys. A 638, 195C (1998).

16. J.-Y. Ollitrault, Phys. Rev. D 46, 229 (1992).

17. J. Barrette et al. [E877 Collaboration], Phys. Rev. C 56, 3254 (1997).

18. H. Appelshäuser et al. [NA49 Collaboration], Phys. Rev. Lett. 80, 4136 (1998).

19. P. F. Kolb, U. W. Heinz, P. Huovinen, K. J. Eskola and K. Tuominen, Nucl. Phys. A 696, 197 (2001).

20. S. A. Voloshin and A. M. Poskanzer, Phys. Lett. B 474, 27 (2000). 
21. Z. w. Lin and C. M. Ko, Phys. Rev. C 65, 034904 (2002).

22. D. Molnar and M. Gyulassy, Nucl. Phys. A 697, 495 (2002) [Erratum-ibid. A 703, $893(2002)]$.

23. P. F. Kolb, J. Sollfrank and U. W. Heinz, Phys. Rev. C 62, 054909 (2000); P. Huovinen et al., Phys. Lett. B 503, 58 (2001).

24. C. Adler et al. [STAR Collaboration], Phys. Rev. Lett. 87, 182301 (2001).

25. X. N. Wang, Phys. Rev. C 63, 054902 (2001).

26. P. Danielewicz and G. Odyniec, Phys. Lett. B 157, 146 (1985); A. M. Poskanzer and S. A. Voloshin, Phys. Rev. C 58, 1671 (1998).

27. S. Wang et al., Phys. Rev. C 44, 1091 (1991).

28. P. M. Dinh, N. Borghini and J.-Y. Ollitrault, Phys. Lett. B 477, 51 (2000); Phys. Rev. C 62, 034902 (2000).

29. N. Borghini, P. M. Dinh and J.-Y. Ollitrault, Phys. Rev. C 63, 054906 (2001); Phys. Rev. C 64, 054901 (2001).

30. A. H. Tang (STAR Collaboration), hep-ex/0108029.

31. C. Adler et al. [STAR Collaboration], nucl-ex/0206001.

32. Y. V. Kovchegov and K. L. Tuchin, hep-ph/0203213. 
OLLITRAULT.tex; 23/08/2002; $12: 31 ;$ p.22 